PANCREATITIS

\title{
Echo enhanced ultrasound: a new valid initial imaging approach for severe acute pancreatitis
}

\author{
S Rickes, C Uhle, S Kahl, S Kolfenbach, K Monkemuller, O Effenberger, \\ $P$ Malfertheiner
}

See end of article for authors' affiliations

Correspondence to

Dr S Rickes, Otto-von

Guericke-University

Magdeburg, Department

of Gastroenterology,

Hepatology and Infectious

Diseases, Leipziger Strasse

44, 39120 Magdeburg,

Germany; steffen.rickes@

medizin.uni-magdeburg. de

Accepted 27 June 2005

Published Online first

16 August 2005

Aim: This prospective study aimed to compare the accuracy of echo enhanced ultrasound with spiral computed tomography (CT) in assessing acute pancreatitis and to explore the correlation between ultrasound findings and clinical outcome.

Methods: Thirty one patients (24 men and 7 women, median age 39 years, range 19-67 years) with acute pancreatitis were investigated by contrast enhanced CT and echo enhanced ultrasound within 72 hours after admission. Echo enhanced ultrasound (with intravenous injection of $2.4 \mathrm{ml}$ SonoVue, pulse inversion technique, mechanical index 0.1 to 0.2 , Siemens Elegra) was performed following conventional ultrasound. Balthazar's grading system was used to measure CT and ultrasound severity indices (CTSI and USSI). Correlations between CTSI and USSI and between USSI and clinical parameters were tested by Spearman's rank correlation coefficient.

Results: A strong correlation was demonstrated between CTSI and USSI $(r=0.807, \mathrm{p}<0.01)$. Ultrasound correlated with the following: the Ranson score $(r=0.401, p<0.05), C$-reactive protein levels 48 hours after admission $(r=0.536, p<0.01)$, duration of hospitalisation $(r=0.422, p<0.05)$, and clinical outcome regarding morbidity, including local and systemic complications $(r=0.363, p<0.05)$. Based on CT findings as the gold standard, sensitivity, specificity, positive predictive value, and negative predictive value of ultrasound for detecting severe acute pancreatitis based on imaging criteria (Balthazar score D or $\mathrm{E}$ and/or presence of hypoperfusion compatible with necrosis and/or SI $\geqslant 3$ ) were, respectively, $82 \%$ (95\% $\mathrm{Cl} 61$ to 93 ), 89\% (95\% Cl 57 to 98), 95\% (95\% Cl 75 to 99), and 67\% (95\% Cl 39 to 86).

Conclusion: Echo enhanced ultrasound produces excellent results in the staging of acute pancreatitis severity. The procedure is cheaper and has fewer contraindications than CT. Further multicentre studies need to be performed before including the method in the diagnostic algorithm of patients with acute pancreatitis.

A cute pancreatitis presents with a wide clinical spectrum of complications. Early assessment and differentiation of oedematous acute pancreatitis and necrotising or severe acute pancreatitis allow distinct therapeutic algorithms. ${ }^{12}$ In the majority of patients the disease is relatively mild, with low morbidity and mortality. ${ }^{2}$ However, in 10-25\% of patients the clinical course is severe, requiring intensive care and sometimes surgical or radiological intervention. Thus if early treatment of patients with severe pancreatitis can result in less morbidity and mortality, accurate identification of patients with severe disease is important. ${ }^{3}$

Spiral computed tomography (CT) is currently considered the imaging reference standard for diagnosis and staging of acute pancreatitis. ${ }^{45}$ This procedure allows the detection and grading of pancreatic necrosis and acute fluid collections, and these parameters have been shown to correlate with the course of the disease. ${ }^{6}$ However, this procedure requires intravenous injection of iodinated contrast medium for the detection of hypoperfused areas in the pancreatic parenchyma. Iodinated contrast media are linked with nephrotoxicity, which limits their use in patients with acute renal failure. Furthermore, contrast media have the potential to aggravate acute pancreatitis in animal models by impairing the pancreatic microcirculation. ${ }^{7-9}$ These findings have no proven relevance in humans. ${ }^{10}{ }^{11}$ Nevertheless, an imaging procedure with fewer side effects deserves consideration as a safer alternative.

Conventional transabdominal ultrasound plays only a limited role in the diagnosis of acute pancreatitis. The main problem is that with this procedure a detection of pancreatic necrosis is difficult because it cannot assess organ perfusion.
Furthermore the pancreas may not be visible in all patients because of meteorism and/or abdominal pain. On the other hand, ultrasound often represents the first imaging technique in the evaluation of patients with acute pancreatitis. An increase in pancreas volume or changes in parenchymal structure can be detected. ${ }^{12-18}$ Also, complications like local fluid collections can be diagnosed. Doppler imaging offers valuable information regarding potential vascular complications-for example, thrombosis of the splenic vein. Ultrasound has not only diagnostic value but can also be used for the treatment of complications of acute pancreatitis (such as percutaneous ultrasound guided drainage).

Through the use of echo enhancers, however, even ultrasound can nowadays examine the vascularisation behaviour of the pancreas and liver parenchyma in sufficient detail and in this way it may assist in, for example, tumour differentiation. ${ }^{12-18}$ SonoVue (Bracco International BV, Amsterdam, the Netherlands) is a so-called second generation echo enhancer designed and optimised with regard to the resistance to pressure. It consists of sulfur hexafluoride filled microbubbles which are surrounded by a shell composed of a phospholipidic monolayer. ${ }^{12-18}$ The use of an echo enhancer increases the Doppler signal, which is generated by destroying the microbubbles. Echo enhanced ultrasound employs the so-called pulse inversion process, in which two ultrasonic waves that are $180^{\circ}$ out of phase with

Abbreviations: CTSI, computed tomography severity index; NPV, negative predictive value; PPV, positive predictive value, $\mathrm{Sn}$, sensitivity; $\mathrm{Sp}$, specificity; USSI, ultrasound severity index. 
each other are transmitted into the tissue under examination, one shortly after the other. The ultrasound signals then become quenched in media such as tissue that vibrate linearly, and become enhanced in media such as gas microbubbles that vibrate non-linearly. ${ }^{12-18}$

The aims of the present study were to compare the diagnostic performance of echo enhanced US with CT in the assessment of acute pancreatitis (especially in the detection of pancreatic necrosis), and to explore the correlation between sonographic findings and clinical outcome.

\section{PATIENTS AND METHODS}

\section{Patients}

Forty patients with acute pancreatitis who had been referred to the Department of Gastroenterology of the Otto-vonGuericke-University Magdeburg were prospectively studied from April 2003 to January 2005. The diagnosis of acute pancreatitis was based on the patients' symptoms (such as belt-like epigastric pain, fever, nausea, and vomiting) and on elevation of serum lipase by at least a factor of 3. No patient had a history of a long term chronic pancreatitis. All patients gave their informed consent to participation in the study, and the study protocol was approved by the local ethics committee. Three patients had to be excluded from the study because sonographic imaging of the pancreas was impaired by meteorism, three patients had a contraindication to administration of the ultrasound contrast medium SonoVue on account of coronary heart disease, and three patients were excluded because of contraindications to CT with contrast injection (renal failure or known allergy to iodinated contrast medium). Consequently, the study comprised 31 patients (24 men and seven women, median age 39 years, range 19-67 years). The aetiology of acute pancreatitis was alcohol abuse in 19 patients and gallstones in six. Aetiology remained unidentified in six patients.

\section{Clinical variables}

The following variables were examined to characterise the severity of acute pancreatitis: (a) severity on admission, assessed by clinical examination and laboratory data, patients with local and systemic complications being distinguished in accordance with the Atlanta criteria; ${ }^{19}$ (b) the Ranson score; (c) levels of C-reactive protein in $\mathrm{mg} / \mathrm{dl}$ 48 hours after admission; (d) duration of hospitalisation; and (e) clinical outcome 30 days after admission. This outcome was graded on a scale from 0 to 3, as follows: 0 , complete resolution to normal; 1, local complications (pseudocyst formation); 2, systemic complications (sepsis, organ failure); and 3, death.

\begin{tabular}{|c|c|c|}
\hline & Ultrasound patients & CT patients \\
\hline \multicolumn{3}{|l|}{ Balthazar's grade } \\
\hline A & 8 & 4 \\
\hline B & 4 & 0 \\
\hline C & 1 & 6 \\
\hline D & 9 & 10 \\
\hline $\mathrm{E}$ & 9 & 11 \\
\hline \multicolumn{3}{|l|}{ Necrosis } \\
\hline None & 21 & 23 \\
\hline$<30 \%$ & 3 & 2 \\
\hline $30 \%-50 \%$ & 5 & 4 \\
\hline$>50 \%$ & 2 & 2 \\
\hline \multicolumn{3}{|l|}{ CTSI and USSI } \\
\hline Median (range) & $3(0-10)$ & $3(0-10)$ \\
\hline
\end{tabular}
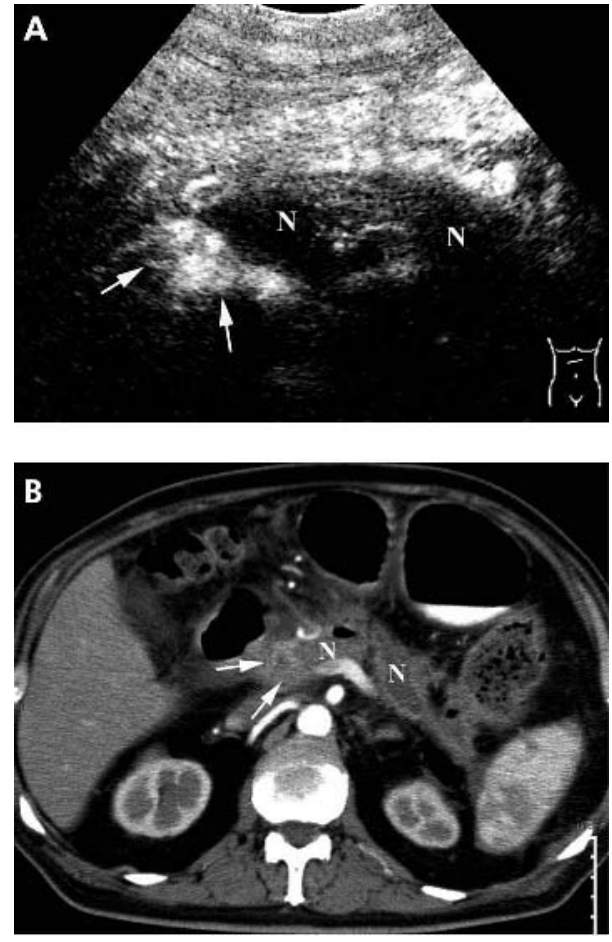

Figure 1 Severe necrotising acute pancreatitis in ultrasound (A) and CT (B), 20 hours after admission. (A) Detection of echo poor areas in the body and tail of the pancreas (N), which show no vascularisation in echo enhanced ultrasound and so correspond to necroses. In contrast, part of the undamaged parenchyma of the head of the pancreas shows good perfusion (arrows). (B) CT performed directly after ultrasound and showing necrotic (N) and vital (arrows) areas of the pancreas.

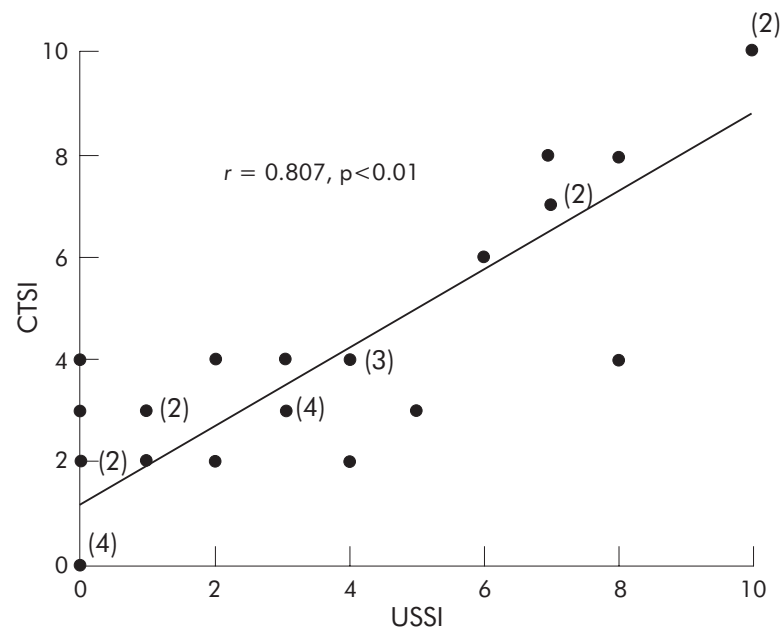

Figure 2 Spearman's correlation between ultrasound (USSI) and computed tomography severity indices (CTSI) in 31 patients with acute pancreatitis. Numbers in parentheses indicate the number of patients represented by the adjoining square.

\section{Ultrasound techniques}

Within 72 hours after admission, all patients were investigated by an experienced examiner blinded for other laboratory and imaging findings. A dynamic 2-5 MHz sector scanner (Siemens Elegra, Erlangen, Germany) was used. All investigations were done at the ultrasound laboratory of the 
Department of Gastroenterology. First the entire pancreas and the peripancreatic tissue were examined transabdominally using conventional B-mode ultrasound. In some patients with obesity tissue harmonic imaging was used for optimising the image quality. ${ }^{20}$ Thrombosis of adjacent vessels were excluded by colour and/or power Doppler technique. Echo enhanced ultrasound of the pancreatic parenchyma under the conditions of pulse inversion imaging was then started immediately after peripheral intravenous injection of $2.4 \mathrm{ml}$ SonoVue. A mechanical index of 0.1 to 0.2 was used. The investigation lasted approximately two minutes (including the arterial, capillary, and venous phases).

\section{Spiral CT}

Computed tomography (16-line spiral CT, Aquillion, Toshiba, Neuss, Germany) took place within four hours after ultrasound (in all cases within 72 hours after the onset of symptoms). Oral contrasting of the gastrointestinal tract with a Telebrix-water mixture was followed by data acquisition in the pancreatic region using a $16 \times 1 \mathrm{~mm}$ slice thickness, both in the native phase and in the arterial and portal-venous contrast phase (after intravenous injection of $100 \mathrm{ml}$ Imeron 300 (Altana, Konstanz, Germany)). Hence axial, coronary, and sagittal single images with slice thicknesses of $1 \mathrm{~mm}$ and $5 \mathrm{~mm}$ were calculated at reconstruction intervals of $0.8 \mathrm{~mm}$ and $4 \mathrm{~mm}$ respectively.

\section{Image analysis}

Computed tomography and ultrasound data were analysed separately and immediately after the procedure at the work station, each by experienced examiners (SR for ultrasound, and $\mathrm{OE}$ for $\mathrm{CT}$ ), who were blinded to the clinical and biological settings and to the results of the related imaging procedure. Imaging findings were recorded on standardised data collection sheets on a central server and/or videotapes (for ultrasound), and included the following: (1) the presence, extent, and content of pancreatic and peripancreatic fluid collections; (2) the presence of necrosis of the pancreatic parenchyma. For ultrasound, pancreatic necrosis was defined as the detection of a liquid area in conventional $\mathrm{B}$-mode or evidence of an echo poor region in conventional B-mode with loss of signal enhancement after injection of the echo enhancer. For CT it was defined as an area of pancreatic parenchyma with loss of contrast enhancement after injection of the iodinated contrast medium. Acute pancreatitis

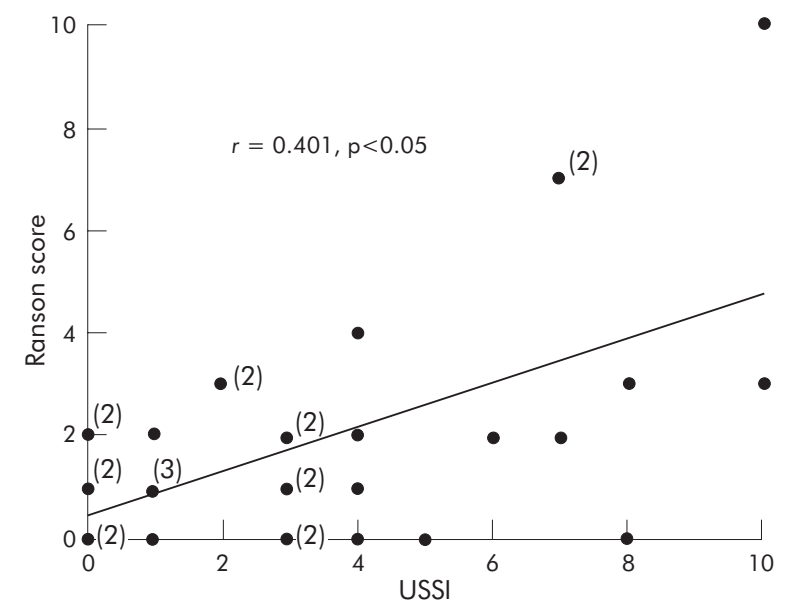

Figure 3 Spearman's correlation between ultrasound severity index (USSI) and the Ranson score for 31 patients with acute pancreatitis. Numbers in parentheses indicate the number of patients represented by the adjoining square. severity was graded according to Balthazar's criteria for the extent of pancreatic and extrapancreatic fluid collections, degree of necrosis, and the combined severity index. ${ }^{6}$ The severity index previously described for CT (CTSI) was similarly established for echo enhanced ultrasound (USSI).

\section{Statistical analysis}

Descriptive statistics were expressed as the median and range for continuous variables. Percentage values are rounded to the nearest whole number. Correlations between USSI and CTSI and between USSI and clinical parameters (Ranson score, CRP levels, clinical outcome, and length of hospital stay) were tested by Spearman's rank correlation coefficient. Taking CT as gold standard, we calculated the sensitivity $(\mathrm{Sn})$, specificity $(\mathrm{Sp})$, positive predictive value (PPV), and negative predictive value (NPV) of ultrasound in detecting severe acute pancreatitis, based on imaging features (Balthazar score D or E and/or presence of hypoperfusion compatible with necrosis and/or SI $\geqslant 3$ ). The $95 \%$ confidence intervals (CI) were calculated for these values. Moreover, we calculated the same parameters of ultrasound diagnostic accuracy (Sn, Sp, PPV, NPV) for detecting severe acute pancreatitis, but taking the Ranson score as gold standard ( severe acute pancreatitis defined as a Ranson score $\geqslant 3$ ). For comparisons between conventional (without injection of an echo enhancer) and echo enhanced ultrasound, McNemar's test was used. p values of less than 0.05 were considered significant. Data analysis was done with SPSS, version 10.0 (SPSS Inc, Chicago, IL, USA).

\section{RESULTS}

\section{Clinical and laboratory findings}

On admission, all patients were assessed by clinical examination and laboratory data. Six patients (19\%) had systemic complications meeting the Atlanta criteria. The Ranson score was $\geqslant 3$ for eight patients $(26 \%)$, and the median Ranson score was $1(0-10)$. The median C-reactive protein value 48 hours after admission was $11 \mathrm{mg} / \mathrm{dl}(0-41)$. C-reactive protein levels 48 hours after admission were $>10 \mathrm{mg} / \mathrm{dl}$ in 21 patients $(68 \%)$. The median length of hospitalisation was 13 days (range 4-39).

\section{Comparison between $\mathrm{CT}$ and ultrasound findings}

Balthazar's acute pancreatitis severity grade, denoting the extent of pancreatic and extrapancreatic acute fluid collections, and the degree of necrosis on CT and ultrasound on admission are shown for all 31 patients in table 1. Eight $(26 \%)$ patients had pancreatic necrosis at CT. All necroses were diagnosed correctly by echo enhanced ultrasound (fig l). However, with this procedure there were two false positive results which corresponded to fibrosis and small pseudocysts at CT. With conventional ultrasound (without injection of an echo enhancer), necrosis was detectable in only two $(6 \%)$ of the 31 cases $(p<0.05)$; in the remaining cases this procedure could not distinguish between parenchymal necrosis and oedema.

\begin{tabular}{|c|c|}
\hline Parameters & $\mathrm{CT} v$ ultrasound \\
\hline $\begin{array}{l}\text { Severity index } \\
\text { Balthazar's grade } \\
\text { Extent of necrosis }\end{array}$ & $\begin{array}{l}r=0.807^{*} \\
r=0.721^{*} \\
r=0.885^{*}\end{array}$ \\
\hline
\end{tabular}




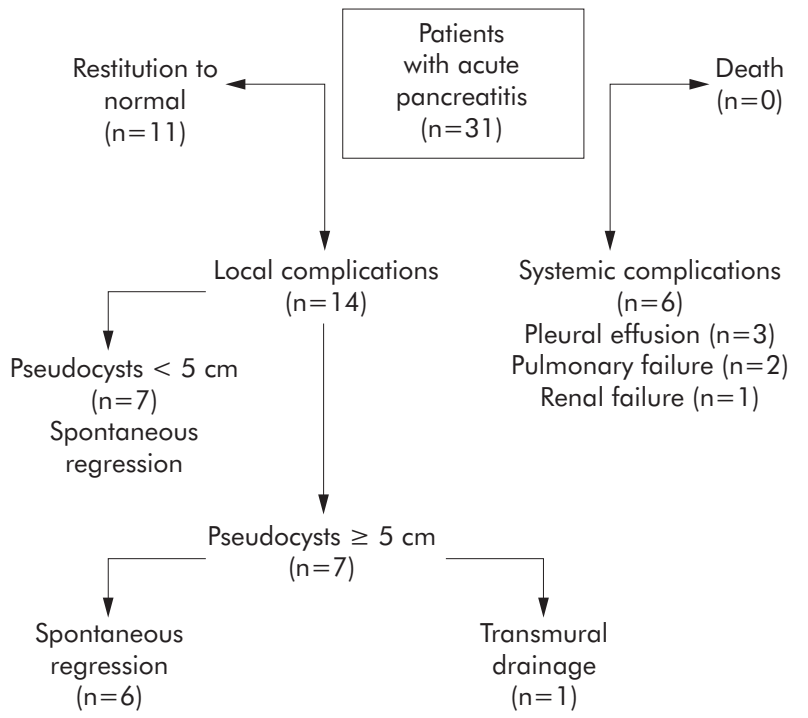

Figure 4 Clinical outcome of 31 patients with acute pancreatitis 30 days after admission.

A significant correlation was found between the two severity indices (CTSI and USSI) $(r=0.807, \mathrm{p}<0.01$; fig 2 and table 2). Furthermore, comparison of the results of CT and ultrasound for the spread of acute fluid collections (Balthazar's grade) and the extent of necrosis revealed a significant correlation between the two imaging techniques (table 2).

Taking CT as the gold standard, Sn, Sp, PPV, and NPV of ultrasound for detecting severe acute pancreatitis based on imaging criteria (Balthazar score D or E and/or presence of hypoperfusion compatible with necrosis and/or SI $\geqslant 3$ ) were respectively $82 \%$ (95\% CI 61 to 93 ), $89 \%$ (95\% CI 57 to 98 ), $95 \%$ (95\% CI 75 to 99 ), and $67 \%$ (95\% CI 39 to 86 ).

\section{Comparison between ultrasound and clinical variables and outcome}

A significant correlation was observed between USSI on the one hand and the Ranson score $(r=0.401, \mathrm{p}<0.05$; fig 3$)$, CRP levels 48 hours after admission $(r=0.536, \mathrm{p}<0.01)$, clinical outcome $(r=0.363, \mathrm{p}<0.05)$, and length of hospital stay $(r=0.422, \mathrm{p}<0.05)$ on the other.

Finally, taking the Ranson score as reference standard (severe acute pancreatitis defined as a Ranson score $\geqslant 3$ ), ultrasound detected severe acute pancreatitis with an Sn, Sp, PPV, and NPV of respectively 88\% (95\% CI 53 to 98 ), $70 \%$ (95\% CI 50 to 84 ), $50 \%$ (95\% CI 27 to 73 ), and $94 \%$ (95\% CI 73 to 99$)$. Similarly, CT detected severe acute pancreatitis with an Sn, Sp, PPV, and NPV of respectively 88\% (95\% CI 53 to 98 ), $35 \%$ (95\% CI 19 to 55 ), $32 \%$ (95\% CI 16 to 53 ), and $89 \%$ (95\% CI 57 to 98$)$.

Eleven patients (36\%) experienced complete resolution to normal without morbidity, 14 (45\%) developed local complications, and six (19\%) developed systemic complications with the need of intensive care treatment (three of the latter had pleural effusion, two had pulmonary failure, and one had renal failure). Seven patients showed pseudocysts exceeding $5 \mathrm{~cm}$ in diameter; spontaneous regression occurred in six and one patient required transmural drainage of a large symptomatic pseudocyst (fig 4).

\section{DISCUSSION}

The present results show that echo enhanced ultrasound has the potential to become a reliable alternative to contrast
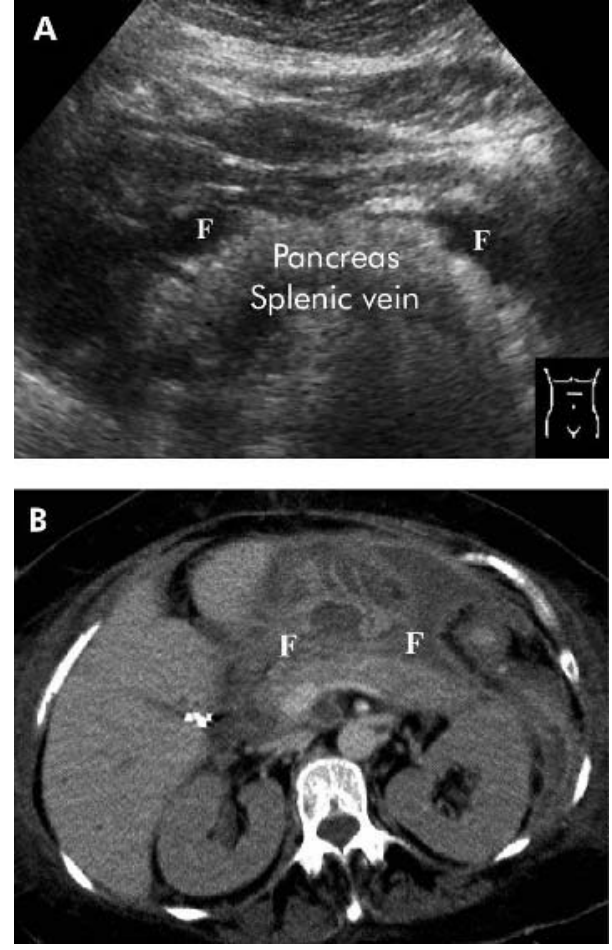

Figure 5 Detection of peripancreatic fluid collection (F) at ultrasound (A) and CT (B), 24 hours after admission.

enhanced CT for assessing the severity of acute pancreatitis and predicting its outcome. For the detection of organ necroses in particular, the additional use of an echo enhancer gave good correlation with CT as gold standard $(r=0.885)$. Conventional ultrasound (without injection of an echo enhancer) is ineffective in the detection of pancreatic necroses. In the present study, necroses were detected in only two out of eight cases with conventional ultrasound but in all eight cases with echo enhanced ultrasound $(p<0.05)$. Differentiation of fibroses and small pseudocysts remains difficult, however, even for echo enhanced ultrasound, leading in the present study to two false positive findings in the detection of organ necroses.

For Balthazar's grade the correlation between ultrasound and CT was somewhat poorer $(r=0.721)$ than for the diagnosis of organ necroses, the main reason being the limitations on sonographic detection of the peripancreatic fatty tissue necrosis (table 2). Whereas CT found six instances of Balthazar's grade C, only one instance was found with echo enhanced ultrasound. Peripancreatic infiltrations can only be detected after cavitation of the tissue, whereupon good agreement regarding the detection of Balthazar's grades D and E is achieved (table 2, fig 5).

Contrast enhanced CT is currently considered the imaging technique of choice for assessment of severity in acute pancreatitis. ${ }^{6}{ }^{21-26}$ However, one of its limitations in very ill patients is the need to inject contrast medium, which can lead to acute renal failure, especially in the presence of hypovolaemia, which is frequent in severe acute pancreatitis. $^{7-9}$ Furthermore, CT is costly (€300/\$364 per scan), and contrast medium has been found to aggravate acute pancreatitis in animal models by impairing pancreatic microcirculation, ${ }^{7-9}$ although these findings have not been confirmed in humans. ${ }^{10} 11$ For these reasons, an imaging procedure with lower costs and fewer side effects could be considered as a safer alternative. In comparison with CT, echo enhanced ultrasound is much cheaper (€35/ $\$ 42$ per scan) 
and has fewer side effects. ${ }^{27}$ In the present study, however, three patients with coronary heart disease had to be excluded from participation on account of a temporary licence restriction for the echo enhancer SonoVue. This licence restriction has now been lifted. Without this restriction, only three patients with pronounced meteorism would have been unexaminable and the proportion of patients requiring exclusion would have been $8 \%$ both for echo enhanced ultrasound and CT. However, at the moment, the use of SonoVue is not permitted in patients with serious pulmonary or cardiac diseases-for example, adult respiratory distress syndrom and cardiac infarction. On the other hand, CT is also operator dependent like other imaging techniques including ultrasound.

We determined severity indices based on CT and ultrasound findings. Significant correlations were observed between CTSI and USSI, as well as between USSI, clinical variables linked to the severity of acute pancreatitis, and clinical outcome.

Taking CT as the gold standard for detecting severe acute pancreatitis, on the basis of imaging features (Balthazar score $\mathrm{D}$ or $\mathrm{E}$ and/or presence of hypoperfusion compatible with necrosis and/or SI $\geqslant 3$ ) ultrasound demonstrated a sensitivity of $82 \%$ and a PPV of $95 \%$. In depth, discordance between the two techniques was observed in five patients (16\%). In four patients ultrasound assessed acute pancreatitis as mild whereas CT evaluated it as severe (false negatives). On the other hand, ultrasound overestimated the severity of acute pancreatitis in one patient (false positive). Interestingly, based on clinical criteria (Ranson score), none of the four false negative patients had severe acute pancreatitis. For the false positive finding the Ranson score was also $<3$, however.

These elements underline the fact that, although CT was taken as the gold standard in acute pancreatitis assessment to determine the diagnostic accuracy of echo enhanced ultrasound, it is not clear whether it is really the reference standard in predicting severe acute pancreatitis for all cases. Moreover, if the Ranson score is taken as the reference standard (acute pancreatitis defined as severe if Ranson score $\geqslant 3$ ), Sn values for the two imaging techniques are identical, a fact that further questions the superiority of CT. However, it must be borne in mind that pathological lesions in acute pancreatitis can possibly be diagnosed at an earlier stage with CT than with ultrasound and the laboratory data, and this would explain the relatively low specificity of CT in correlation with the Ranson score. One potential limitation of our study regarding the evaluation of the diagnostic accuracy of the two techniques is the large $95 \%$ confidence intervals, which could result from the relatively small sample size. However, in our study we included a similar number of patients as other studies designed to evaluate the usefulness of new imaging procedures in severe acute pancreatitis. ${ }^{21}$

In conclusion, echo enhanced ultrasound is comparable to contrast enhanced CT for the assessment of patients with acute pancreatitis and can be recommended as a first choice imaging procedure, especially when iodinated contrast medium injection is contraindicated. It can accurately determine severity and predict clinical outcome during the course of acute pancreatitis. However, the impact of these findings on acute pancreatitis assessment and management should be further investigated.

\section{Authors' affiliations}

S Rickes, C Uhle, S Kahl, S Kolfenbach, K Monkemuller,

P Malfertheiner, Department of Gastroenterology, Hepatology and Infectious Diseases, Otto-von-Guericke-University, Magdeburg, Germany

O Effenberger, Department of Radiology, Otto-von-Guericke-University, Magdeburg, Germany

Competing interest: none declared.

\section{REFERENCES}

1 Sarner M, Cotton PB. Classification of pancreatitis. Gut 1984;25:756-9.

2 Bradley III EL. The necessity for a clinical classification of acute pancreatitis: the Atlanta system. In: Bradley EL III, eds. Acute pancreatitis, diagnosis and therapy. New York: Raven Press, 1994:53-62.

3 Poston GJ, Williamson RCN. Surgical management of acute pancreatitis. Br J Surg 1990;77:5-12.

4 Balthazar EJ. Acute pancreatitis: assessment of severity with clinical and CT evaluation. Radiology 2002;223:603-13.

5 Banks PA. Practice guidelines in acute pancreatitis. Am J Gastroenterol 1997:92:377-86.

6 Balthazar EJ, Robinson DL, Megibow AJ, et al. Acute pancreatitis: value of CT in establishing diagnosis. Radiology 1990;174:331-6.

7 Foitzik T, Bassi DG, Schmidt J, et al. Intravenous contrast medium accentuates the severity of acute necrotizing pancreatitis in the rat. Gastroenterology 1994; 106:207-14.

8 Foitzik T, Bassi DG, Fernandez-del Castillo C. Intravenous contrast medium impairs oxygenation of the pancreas in acute necrotizing pancreatitis in the rat. Arch Surg 1994; 129:706-11.

9 Schmidt J, Hotz HJ, Foitzik T, et al. Intravenous contrast medium aggravates the impairment of pancreatic microcirculation in necrotizing pancreatitis in the rat. Ann Surg 1995;221:257-64.

10 Carmona-Sanchez R, Uscanga L, Bezaury-Rivas P, et al. Potential harmful effect of iodinated intravenous contrast medium on the clinical course of mild acute pancreatitis. Arch Surg 2000;135:1280-4.

11 Uhl W, Roggo A, Kirschstein T, et al. Influence of contrast-enhanced computed tomography on course and outcome in patients with acute pancreatitis. Pancreas 2002;24:191-7.

12 Rickes S, Unkrodt K, Ocran K, et al. Differentiation of neuroendocrine tumours from other pancreatic lesions by echo-enhanced power Doppler sonography and somatostatin receptor scintigraphy. Pancreas 2003;26:76-81.

13 Rickes S, Unkrodt K, Neye H, et al. Differentiation of pancreatic tumours by conventional ultrasound, unenhanced and echo-enhanced power Doppler sonography. Scand J Gastroenterol 2002;37:1313-20.

14 Rickes S, Wermke W. Differentiation of cystic pancreatic neoplasms and pseudocysts by conventional and echo-enhanced ultrasound. J Gastroenterol Hepatol 2004;19:761-6.

15 Rickes S, Schulze S, Neye H, et al. Improved diagnosing of small hepatocellular carcinomas by echo-enhanced power Doppler sonography in patients with cirrhosis. Eur J Gastroenterol Hepatol 2003;15:893-900.

16 Schlottmann K, Kratzer W, Schölmerich J. Doppler ultrasound and intravenous contrast agents in gastrointestinal tract disorders: current role and future implications. Eur J Gastroenterol Hepatol 2005; 17:263-75.

17 Rickes S, Ocran K, Schulze S, et al. Evaluation of Doppler sonographic criteria for the differentiation of hepatocellular carcinomas and regenerative nodules in patients with liver cirrhosis. Ultraschall Med 2002;23:83-90.

18 Rickes S, Unkrodt K, Ocran K, et al. Evaluation of doppler ultrasonography criteria for the differential diagnosis of pancreatic tumours. Ultraschall Med 2000;20:253-8.

19 Bradley EL. A clinically based classification system for acute pancreatitis. Arch Surg 1993; 128:586-90

20 Garg M, Sandhu M, Sood B, et al. Tissue harmonic imaging in the evaluation of acute pancreatitis. Australas Radiol 2004;48:117-22.

21 Arvanitakis $M$, Delhaye $M$, De Maertelaere V, et al. Computed tomography and magnetic resonance imaging in the assessment of acute pancreatitis. Gastroenterology 2004;126:715-23.

22 Baron TH, Morgan DE. Acute necrotizing pancreatitis. N Engl J Med 1999;340:1412-17.

23 Vege SS, Baron TH. Management of pancreatic necrosis in severe acute pancreatitis. Clin Gastroenterol Hepatol 2005;3:192-6.

24 Uhl W, Warshaw A, Imrie C, et al. IAP Guidelines for the surgical management of acute pancreatitis. Pancreatology 2002;2:565-73.

25 Raraty MG, Connor S, Criddle DN, et al. Acute pancreatitis and organ failure: pathophysiology, natural history, and management strategies. Curr Gastroenterol Rep 2004;6:99-103.

26 Shankar S, van Sonnenberg E, Silverman SG, et al. Imaging and percutaneous management of acute complicated pancreatitis. Cardiovasc Intervent Radiol 2004;27:567-80.

27 Albrecht T, Blomley M, Bolondi L, et al. Guidelines for the use of contrast agents in ultrasound. Ultraschall Med 2004;25:249-56. 